\title{
RADIAL ELECTRIC FIELDS AND TRANSPORT BARRIERS
}

\author{
Maarten Vergote, Kristel Crombé \\ Laboratory for Plasma Physics, Association 'EURATOM-Belgian State' \\ Ecole Royale Militaire - Koninklijke Militaire School, Avenue de la Renaissance 30, B-1000 Brussels, Belgium
}

\section{INTRODUCTION}

The importance of radial (i.e. perpendicular to the magnetic surface) electric fields was already recognised early in the research on controlled thermonuclear fusion. An initial description of electric field effects in toroidal confinement was given by Budker[6]. Such a configuration with combined magnetic and electric confinement ("magnetoelectric confinement", where the electric field provides a toroidal equilibrium configuration without rotational transform) was studied by Stix[7], who suggested that a reactor-grade plasma under magnetoelectric confinement (electric fields of order $1 \mathrm{MV} / \mathrm{cm}$ ) may reach a quasi-steady-state with ambipolar loss of electrons and some suprathermal ions (e.g. $3.5 \mathrm{MeV} \alpha-$ particles). Experiments such as on the Electric Field Bumpy Torus EFBT[8, 9] provided quite favourable scaling for particle confinement. The possible importance of radial electric fields for transport was in the past repeatedly established $[10,11,12,13]$. Since the early days the plasma potential has been measured in tokamaks such as ST[14], TM-4[15] and ISX-B[16], but because no significant effects of the radial electric field $\mathrm{E}_{r}$ on plasma transport were observed under the machine conditions at that time, no further research was conducted in tokamaks.

However, a renaissance came after the transition from a low confinement mode (L-mode) to a high confinement mode (H-mode) was discovered in ASDEX[17]. The interest was suddenly refreshed and a flurry of activity started with the experimental[18, 19] and theoretical recognition[20, 21, 22] of a possible link between $\mathrm{E}_{r}$ and the H-mode phenomenon. Since then research on $\mathrm{E}_{r}$ has flourished and the H-mode has now been seen in a wide variety of magnetic confinement devices. Many theories have pointed to the possible decisive role of $\mathrm{E}_{r}$ in the creation of transport barriers (i.e. zones of finite radial extent where particle and/or heat diffusivity are depressed) and in the L-H bifurcation mechanism.

Typical features of an L-H transition could also be obtained by externally inducing a controlled radial electric field in the plasma (independently of other plasma parameters) in the tokamaks CCT[18] and TEXTOR[23, 24] and later in many other machines [see e.g. reviews[25, 26]]. These electrode biasing experiments (induced H-modes) have con- tributed significantly to the understanding of the $\mathrm{H}$ mode phenomenon and of the effects of $\mathrm{E}_{r}$ on plasma transport[27].

Besides an important theoretical activity, many experiments have since been performed in the plasma edge and the SOL of limiter or divertor devices[25, 26]. Imposing electric fields independently of other machine parameters allows to manipulate the edge and SOL profiles and flows, to control impurities and to affect particle and power exhaust[25].

Radial electric fields have been studied in a variety of devices: tokamaks, stellarators and other helical devices, reversed field pinches, mirrors, etc. In stellarators[28] where neoclassical transport dominates, the transport coefficients depend on $\mathrm{E}_{r}$. A radial electric field limits the excursions of the helically trapped particles due to $\mathbf{E} \times \mathbf{B}$ poloidal rotation, whereby neoclassical transport can be reduced to such an extent that stellarators become viable for a fusion reactor. The present paper concentrates on tokamaks in which $\mathrm{E}_{r}$ itself without shear cannot contribute to confinement improvement because the ensuing rigid rotation which reduces orbit losses ("orbit squeezing") and improves neoclassical transport has no effect on microturbulence which is regarded as the dominating cause of anomalous transport in auxiliary heated tokamaks. Effects of $\mathrm{E}_{r}$ on transport enter only through derivatives of $\mathrm{E}_{r}$.

This paper is mainly based on a former version of this lecture [1], from which the main role of the radial electric field in a tokamak is taken. The underlying mechanism on how it suppresses the turbulent transport is still believed to happen in the way proposed by Burrell[27]. The development of this $\mathbf{E} \times \mathbf{B}$ velocity shear turbulence stabilisation model to explain the formation of transport barriers in magnetic confinement devices is exactly one of the scientific success stories of fusion research. This model has the universality needed to explain turbulence reduction and confinement improvement under a variety of conditions in limiter- and divertor tokamaks, stellarators, torsatrons, reversed field pinches, mirror machines, etc.

Further details on radial electric fields and their role in plasma confinement and exhaust can be found in review articles[29]. 


\section{RADIAL ELECTRIC FIELDS AND ROTATION}

The mechanism of a radial electric field as a transport barrier can be summarized through the link with the $\mathrm{E} \times \mathrm{B}$ drift velocity, creating steady state or oscillatory rotational flows in the poloidal and/or toroidal direction. The correct derivation of this link is described in the framework of neoclassical theory (see [2]). In the core plasma, this theory predicts a link between the radial electric field and (mostly) the toroidal rotation on the basis of ambipolarity, whereas the poloidal flow is strongly damped. In the edge of a tokamak, close to the separatrix, strong gadients in $n$ (and to less extent $T$ ) make that the socalled "anomalous" transport overrules the "theoretical" one predicted by neoclassical theory [3]. In that region other mechanisms come into play; the neoclassical expression for the radial electric field is not valid and it should be replaced by numerical results from codes like ASCOT [4] or B2SOLPS [5].
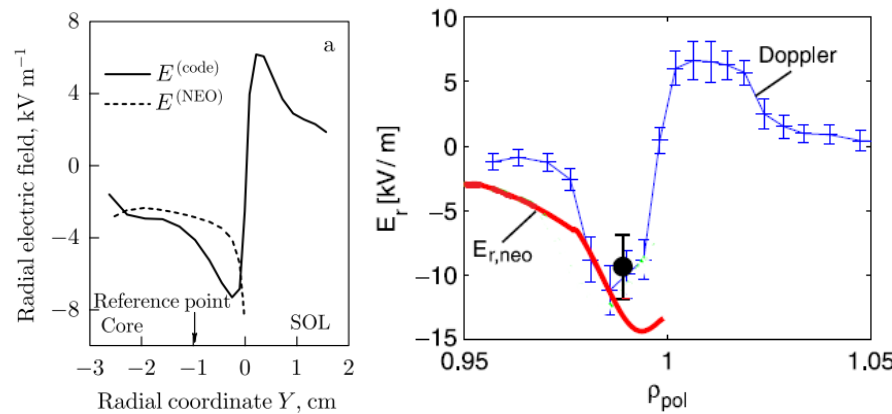

Figure 1: Left: $\mathrm{E}_{r}$ computed in the midplane by B2SOLPS and based on neoclassical theory (taken from [3]). Right: Comparison of $\mathrm{E}_{r}$-measurement in ASDEX Upgrade with $\mathrm{E}_{r, n e o}$ : Doppler reflectometry profile (\#24812, $2.7 \mathrm{~s}$ ) and single point (\#24906, 2.3 $\mathrm{s}$, circle), $\mathrm{E}_{r, \text { neo }}(\# 24906,2.3 \mathrm{~s}$, red), taken from [42]

For every family of species individually, the radial electric field and plasma rotation are connected through the radial momentum balance. From an experimental point of view this opens quite some opportunities, because $\mathrm{E}_{r}$ can be determined from a single (impurity) ion radial force balance equation:

$$
E_{r}=\frac{1}{n_{i} Z_{i} e} \nabla P_{i}-v_{\theta, i} B_{\phi}+v_{\phi, i} B_{\theta}
$$

where $\mathrm{n}_{i}$ is the ion density, $\mathrm{Z}_{i}$ is the charge number of the ion, e is the electronic charge, $\mathrm{P}_{i}$ is the ion pressure, $\mathrm{v}_{\theta i}$ and $\mathrm{v}_{\phi i}$ are the poloidal and toroidal rotation velocities, respectively, of the ion species considered; and $\mathrm{B}_{\theta}$ and $\mathrm{B}_{\phi}$ are the poloidal and toroidal magnetic fields, respectively. This equation is valid at each point on any given flux surface, and the quantities involved are local quantities $\left(\mathrm{E}_{r}\right.$ itself is not a flux function).

It follows from Eq. (1) that $\mathrm{E}_{r}$ is determined by three major driving forces: radial pressure gradient, poloidal and toroidal rotation. Because $\mathrm{E}_{r}$ can be influenced by particle-, heat- and angular momentum input, and by changing the current profile (changing $\mathrm{B}_{\theta}$ ), various of these terms can be active in various machines with respect to $\mathbf{E} \times \mathbf{B}$ shear flow reduction of turbulence and transport, which occurs regardless of the plasma rotation direction. This provides the possibility of active control of transport; $\mathbf{E} \times \mathbf{B}$ shear as a control mechanism for turbulence and transport has the major advantage of flexibility, in that the shear can be generated or enhanced in several ways. Particle-, heat-, and momentum transport are not independent of each other, but have a complex coupling. Therefore, research on $\mathrm{E}_{r}$ can clarify complex plasma transport mechanisms.

\section{E $\times$ B VELOCITY SHEAR REDUCTION OF TURBULENCE}

$\mathbf{E} \times \mathbf{B}$ velocity shear reduction of turbulence in a plasma is a mechanism akin to the interaction between sheared velocity fields and turbulence in fluids. However, in a plasma $\mathbf{E} \times \mathbf{B}$ velocity and fluid velocity due to $\mathrm{E}_{r}$ can be quite different. The fundamental velocity is not the mass velocity, but rather the $\mathbf{E} \times \mathbf{B}$ velocity, the drift velocity at which all particles move - regardless of their charge or mass - and at which turbulent eddies are convected.

The fundamental physics involved in transport reduction is the effect of $\mathbf{E} \times \mathbf{B}$ shear on the growth, radial extent and phase decorrelation of the turbulent eddies. The identification of individual modes responsible for the observed turbulence may not be as important as the knowledge of turbulence drive suppression mechanisms, which provide a direct route to transport control.

Turbulence is stabilised by the shear rate $\omega_{E \times B}$ in the $\mathbf{E} \times \mathbf{B}$ flow velocity $\mathrm{v}_{E \times B}$ induced by $\mathrm{E}_{r}[30]$

$$
\omega_{E \times B}=\left|\frac{d v_{E \times B}}{d r}\right|=\left|\frac{\left(R B_{\theta}\right)^{2}}{B} \frac{d}{d \psi}\left(\frac{E_{r}}{R B_{\theta}}\right)\right|
$$

where $\mathrm{R}$ is the major radius, $\mathrm{B}_{\theta}$ is the poloidal magnetic field and $\psi$ is the poloidal flux.

The $\mathbf{E} \times \mathbf{B}$ shear rate enters quadratically into the various theories; accordingly, its sign is irrelevant. Indeed, H-mode edge barriers have been seen with both signs of $\mathrm{E}_{r}$ and its derivative[33]. Equation (2) shows that both $\mathrm{E}_{r}$ and $\mathrm{B}_{\theta}$ contribute to the final result; $\mathrm{E}_{r} / \mathrm{RB}_{\theta}$ is the toroidal angular speed due to the equilibrium flow driven by $\mathrm{E}_{r}$ in standard neoclassical theory, suggesting that the basic shearing is in the toroidal direction.

Equation (2) also shows that the shear rate is not constant on a given magnetic flux surface, being significantly larger on the low toroidal field side, where the flux surfaces are more dense (the electric potential being constant on a flux surface). Experimental 
data on H-modes have indeed demonstrated significant poloidal variation in the effect of $\mathbf{E} \times \mathbf{B}$ shear on turbulence.

Theoretically, there are two points of view[27]. The first (non-linear suppression) is that the turbulent eddies are distorted and the radial transport is reduced if the $\mathbf{E} \times \mathbf{B}$ shear rate exceeds the decorrelation rate of the ambient turbulence in the absence of $\mathbf{E} \times \mathbf{B}$ shear; this is valid for entire classes of turbulent modes. The second is linear stabilisation, which is mode specific, and therefore the details depend on the turbulence driving mechanisms. The fluctuation spectra are $\mathbf{E} \times \mathbf{B}$ Doppler-shifted, and the stabilisation is mainly due to shear in this Doppler shift.

An important point in plasmas is the synergistic effects between $\mathbf{E} \times \mathbf{B}$ velocity shear and magnetic shear. In neutral fluid dynamics sheared velocity is a source of free energy which can drive turbulence through Kelvin-Helmholtz instabilities. In a plasma shear in the magnetic field prevents coupling of the various modes across the velocity gradient so that they are unable to extract energy from the $\mathbf{E} \times \mathbf{B}$ velocity shear and grow[27].

\section{MEASUREMENT TECHNIQUES}

Different methods exist for measuring the radial electric field in plasmas[34].

A. Spectroscopic measurements by charge exchange recombination spectroscopy (CXRS) [35]:

The different terms in eq. (1) can be measured on impurity ions. A beam of neutral particles (typical deuterium, hydrogen or helium atoms) is injected in the plasma. In some devices a special diagnostic beam is installed for this purpose or alternatively one of the heating beams can be used. Some of the injected neutral atoms transfer an electron to impurity ions. The emitted photons from the impurities in excited state are detected by a spectrometer. From the width, height and Doppler shift of the spectral line, the impurity temperature and density (and thus the pressure) can be calculated, as well as the rotation velocity. Careful attention is required to the correct interpretation of the measured line shape, moreover due to the energy dependence of the charge exchange cross-section and the gyro-orbit motion of the excited ions.

B. Measurements of the perpendicular fluctuation velocity by Doppler reflectometry:

A probing beam is launched at an oblique incidence with respect to the cut-off layer. The backscattered field close to the cut-off layer is detected. Fluctuations whose wave-number $\left(k_{f}\right)$ matches the Bragg rule $k_{f}=-2 k_{i}$ where $k_{i}$ is the probing wavevector at the cut-off, are selected. Since they are aligned with the magnetic field lines $\left(k_{/ /}<<k_{\perp}\right)$ the signal frequency spectrum is Doppler shifted by $\Delta \omega=k_{\perp} \mathrm{v}_{\perp}$, hence allowing the determination of the fluctuation rotation component in the perpendicular direction. $\mathrm{A} \mathrm{v}_{\perp}$ profile is obtained by scanning the probing frequency. The measured velocity $\left(\mathrm{v}_{\perp}\right)$ is the sum of $\mathbf{E} \times \mathbf{B}$ velocity $\left(\mathrm{v}_{E \times B}\right)$ and turbulent phase velocity $\left(\mathrm{v}_{p h}\right)$. When $\mathrm{v}_{p h}$ is much smaller than $\mathrm{v}_{E \times B}$ (which is the normal situation) a direct measurement of $\mathrm{v}_{E \times B}=E_{r} \times B / B^{2}$ is obtained, and thus of the radial electric field when the $B$-field is known.

C. Plasma potential measurements with Heavy Ion Beam Probes (HIBP)[36]:

Single charged particles are generated in an ion source, accelerated in a tube and injected across the magnetic field into the plasma. As particles pass through the plasma they are further ionised to produce double charge exchange particles. The energy of the secondary beam is detected at the energy analyser. An advanced Heavy Ion Beam Probe can simultaneously measure the plasma electric potential $\phi$ (from the difference in energy between the secondary ions leaving the plasma and the primary ions), the electron density $\mathrm{n}_{e}$ (from the intensity of the secondary beam) and its fluctuations, the electron temperature $\mathrm{T}_{e}$, and a poloidal magnetic field component $\mathrm{B}_{\theta}$ at a point inside the plasma. This point can be scanned through the plasma cross-section by varying the deflection potentials (active beam control).

D. Measurements of the plasma potential in the edge region with Langmuir probes[37]:

Langmuir probes can provide radial profiles of $\mathrm{n}_{e}, \mathrm{~T}_{e}$, plasma potential and phase velocity of density turbulent fluctuations. The radial electric field profile is computed from the first derivative of the plasma potential. Langmuir probe measurements are restricted to the plasma edge for high-temperature toroidal plasmas, but they have an excellent spatial resolution of less than $1 \mathrm{~mm}$, while the CXRS and HIBP measurements have typical resolutions above 5 $\mathrm{mm}$.

E. Direct measurement of the radial electric field using Motional Stark Effect polarimetry (MSE) [39]:

MSE is a well established technique for measuring the magnetic field pitch angle in tokamaks. By viewing the Stark emission spectrum from two different angles, this technique can also provide local measurements of the plasma radial electric field $\left(\mathrm{E}_{r}\right)$.

\section{TRANSPORT BARRIERS AND CONFINE- MENT IMPROVEMENT}

As outlined in the review paper of Burrell[26] (see also references therein) the $\mathbf{E} \times \mathbf{B}$ shear stabilisation 
model was originally developed to explain the transport barrier formation at the plasma edge at the $\mathrm{L}$ to $\mathrm{H}$ transition. Later, it has been applied to explain the wider edge transport barrier at the $\mathrm{H}$ - to VH- (very high) mode transition moreover seen in DIII-D. Most recently, this model has been applied to the internal transport barriers (ITB) formed in plasmas with modified (negative or optimised) magnetic shear (DIII-D, TFTR, JT-60U, JET, ASDEX Upgrade, Tore Supra, etc), and to plasmas with transport reduction across the whole plasma radius (JT60U and DIII-D).

\section{A. $\mathbf{E} \times \mathbf{B}$ Shear Effects at the Plasma Edge}

A large variety of studies related to the effect of radial electric fields on edge transport barriers (ETBs) exist. A review of $\mathrm{H}$-mode studies over the past 25 years is given in [40]. The paper concentrates on a couple of the recent results on different tokamaks: ASDEX-Upgrade[41], JET[38], Alcator C$\operatorname{Mod}[44]$ and the spherical tokamak MAST[46].

On $A S D E X$-Upgrade [41], radial electric field and shear measurements were performed using the Doppler reflectometer system as well as the recently installed toroidal and poloidal edge CXRS system following the $B^{5+}$ ions.

The $\mathrm{E}_{r}$ profile has been measured in different confinement regimes. In L-mode, $\mathrm{E}_{r}$ is small in magnitude and exhibits little shear, while in the ETB of the H-mode a strong, negative $\mathrm{E}_{r}$ well and a localized minimum close to the separatrix $\left(\rho_{\text {pol }}>0.99\right)$ is found. The steepest gradients of the pressure profile are in the inner, negative shear region of the $\mathrm{E}_{r}$ well. The depth of the $\mathrm{E}_{r}$ well is observed to increase dramatically with the confinement of the discharge and the main ion pressure gradient term seems to be the dominant contribution to $\mathrm{E}_{r}$ (figure 2). The $\mathrm{E}_{r}$ profile undergoes a reversal at the plasma edge to become positive in the Scrape-Off-Layer, as is measured in Fig. 1.

Figure 3 shows the minimum of $\mathrm{E}_{r, \text { neo }}$ for the different phases at varying densities. Error bars derived from shifting the $\mathrm{T}_{i}$ profile are given for the L-H transition points. At the $\mathrm{L}-\mathrm{H}$ transition $\mathrm{E}_{r, \text { neo }}$ shows no dependence on the electron density at the pedestal top, $\mathrm{n}_{e, p e d}$. Included in figure 3 are also $\mathrm{E}_{r}$ minimum values derived from Doppler reflectometry for a different set of discharges with comparable parameters. This shows a good agreement between CXRS and Doppler reflectometry for the different discharge mode regimes. The very weak variation of the $\mathrm{L}-\mathrm{H}$ points in $\mathrm{E}_{r, \text { neo }}$ is remarkable and underlines the possible key role of $\mathrm{E}_{r}$ in the L-H threshold.

Refurbishment of the $\underline{J E T}$ edge CXRS diagnostic has resulted in higher quality impurity density profiles than previously, allowing analysis of the local $C^{6+}$ impurity ion profiles across the L-H transition. Also with the JET-ITER-Like-Wall a shallow edge radial electric field well is observed at the L-H transi-
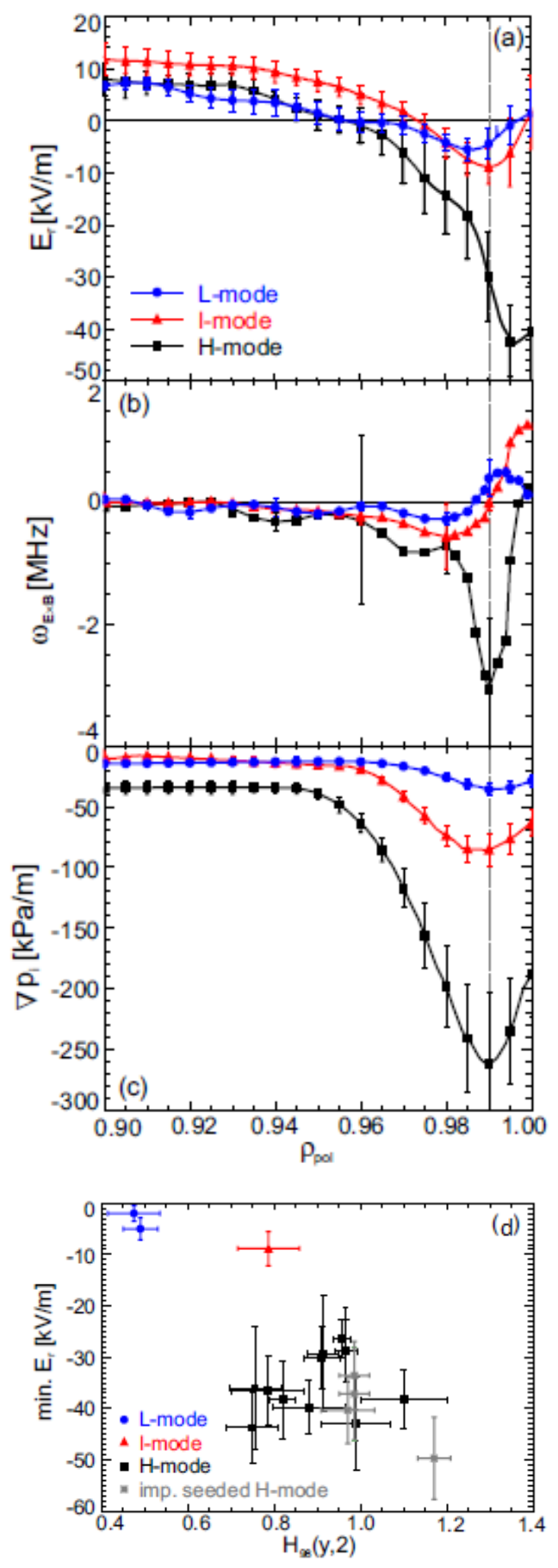

Figure 2: (a) $\mathrm{E}_{r}$ in L-, I- and H-mode and (b) resulting $\mathrm{E}_{r}$ shear. For better clarity the uncertainties are only shown for distinct radial positions. (c) Main ion pressure gradient, $\nabla p_{i}$, in different confinement regimes. (d) Depth of $\mathrm{E}_{r}$ well as a function of the energy confinement factor H98 $(\mathrm{y}, 2)$ [41] 


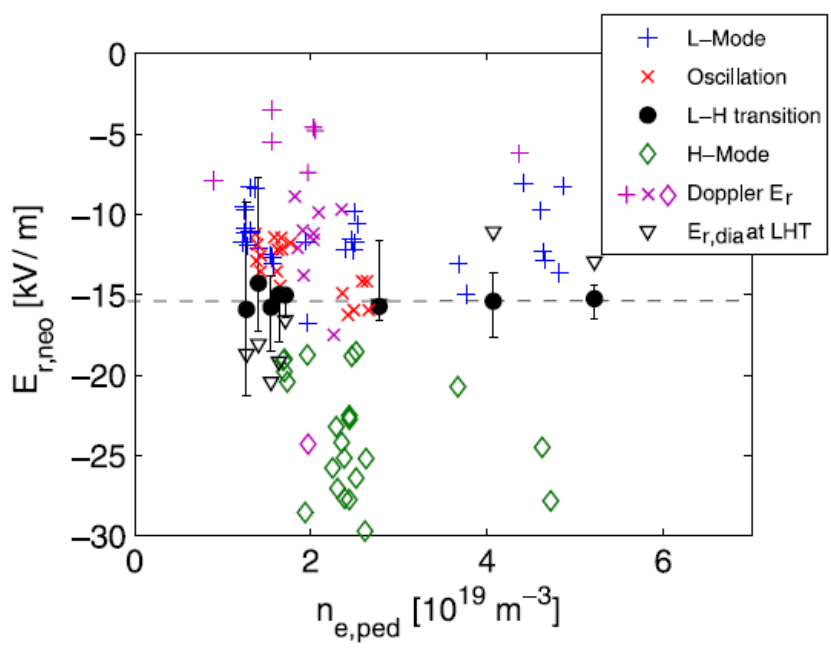

Figure 3: Minimum values in the profile of $\mathrm{E}_{r, \text { neo }}$ versus $\mathrm{n}_{e, p e d}$, Doppler $\mathrm{E}_{r}$ and $\mathrm{E}_{r, d i a}$ for $\mathrm{L}-\mathrm{H}$ transitions only. (figure taken from[42]).

tion. Consistent with previous poloidal velocity measurements in JET, but in contrast with results from other tokamaks, the edge impurity ion poloidal velocity remains low, close to its L-mode values $(0-5$ $\mathrm{km} / \mathrm{s} \pm 2-3 \mathrm{~km} / \mathrm{s}$ ), through the L-H transition and into the ELMy H-mode phase, with no measureable increase within the experimental uncertainties.

The experimental uncertainty in $v_{p o l}$ is dominated by poor photon statistics in the near-separatrix region, where the $C^{6+} \mathrm{CX}$ signal is very weak.

The large error bars in $v_{p o l}$, coupled to its low values in JET, prevent the evaluation of the depth of the total $\mathrm{E}_{r}$ well and of the relative strength of diamagnetic versus poloidal velocity terms in the radial force balance of impurity ions (see fig.4). The diamagnetic term of the negative $\mathrm{E}_{r}$ well increases in magnitude across the $\mathrm{L}-\mathrm{H}$ transition and into the $\mathrm{H}-$ mode phase in the radial region where the edge density and temperature transport barriers have formed and thus is likely to be correlated with the formation of the H-mode pedestal at the L-H transition. The edge toroidal rotation profile does not contribute to the depth of the negative $\mathrm{E}_{r}$ well and thus may not be correlated with the formation of the edge transport barrier in JET.

A new high-resolution CXRS system measuring $\mathrm{B}^{5+}$ ions has enabled the determination of the radial electric field in the Alcator C-Mod edge pedestal with high spatial and temporal resolution[44]. During $H$ mode operation, the radial electric field is positive in the core, but forms a negative well up to $-30 \mathrm{kV} / \mathrm{m}$ deep in the region $10 \mathrm{~mm}$ inside of the LCFS. The well is consistently $5 \pm 1.5 \mathrm{~mm}$ in width regardless of the type of $H$-mode or plasma parameters. This well is largely determined by the poloidal velocity and diamagnetic contributions in the $\mathrm{B}^{5+}$ radial force balance equation. The poloidal velocity contribution is
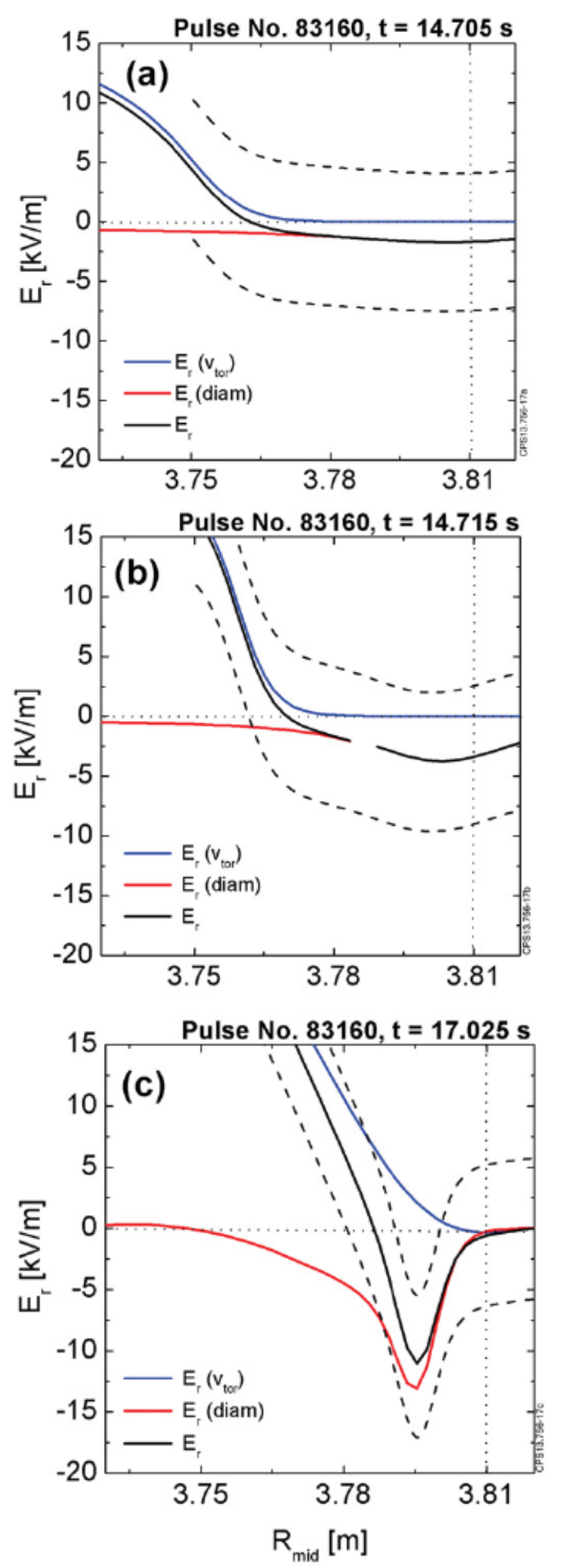

Figure 4: Edge $\mathrm{E}_{r}$ derived from the $C^{6+}$ profiles, and assuming $v_{p o l}=0 \pm 2.5 \mathrm{~km} / \mathrm{s}$ due to the uncertainties. Solid black line: $\mathrm{E}_{r}\left(v_{\text {pol }}=0\right)$; dashed black lines: upper and lower bounds of $\mathrm{E}_{r}$ derived from the uncertainty in the $v_{p o l}$ measurement; solid red line: diamagnetic term; solid blue line: $v_{t o r} \times B_{\text {pol }}$ term. (a) $\mathrm{E}_{r}$ at the $\mathrm{L}-\mathrm{H}$ transition; (b) $\mathrm{E}_{r} 10 \mathrm{~ms}$ after the L-H transition and (c) $\mathrm{E}_{r}$ during the ELMy $\mathrm{H}$-mode phase of the discharge. The vertical dashed lines mark the EFIT separatrix position, $\mathrm{R}_{\text {mid }}$ is the major radius at the magnetic axis, taken from [38] 
typically the larger and narrower contribution of the two and dominates both the $E_{r}$ well width and the $\mathbf{E} \times \mathbf{B}$ shear (see figure 5). The data show a clear correlation between deeper $E_{r}$ wells, higher confinement plasmas, and higher electron temperature pedestal heights. However, improved $L$-mode ( $I$-mode) plasmas exhibit energy confinement equivalent to that observed in similar $H$-mode discharges, but with significantly shallower $E_{r}$ wells. $I$-mode plasmas are characterized by $H$-mode-like energy barriers, but with $L$-mode-like particle barriers. The decoupling of energy and particle barrier formation makes the $I$-mode an interesting regime for fusion research and provides for a low collisionality pedestal without edge localised modes.

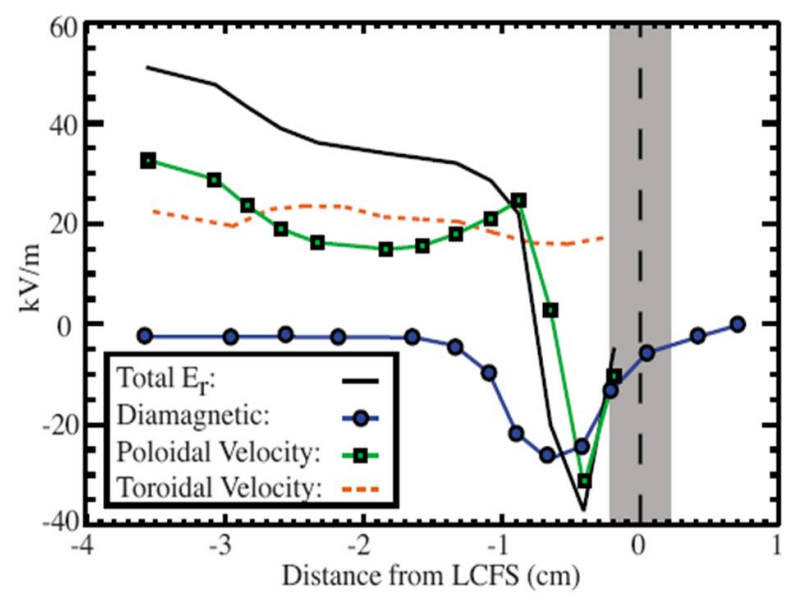

Figure 5: Contributions of the three components from Eq. (1) to the radial electric field in an EDA $H$ mode. The poloidal velocity contribution dominates the width of the $E_{r}$ well (figure taken from[44]).

The first measurements of the structure of the edge radial electric field in a spherical tokamak (MAST) have been presented in[46]. Using active Doppler spectroscopy on $\mathrm{He}^{+}$ with 120 lines of sight $E_{r}$ profiles are calculated from the leading terms of the radial momentum balance equation. A spatial resolution up to $1.5 \mathrm{~mm}$ with a typical time resolution of $5 \mathrm{~ms}$ has been achieved. In L-mode the field is largely determined by the diamagnetic term of the force balance, and fields of only a few $\mathrm{kV} / \mathrm{m}$ are observed. The measured impurity flow is mostly parallel to $\mathbf{B}$, and is greatly affected by MHD, such as sawteeth or mode locking of tearing modes, or error fields. In $\mathrm{H}$-mode a strong perpendicular flow evolves with poloidal and toroidal velocities up to $v_{\phi, \theta}^{\mathrm{He}+} \approx-20 \mathrm{~km} / \mathrm{s}$, and a deep negative electric field well $E_{r}^{\text {min }} \geq-15 \mathrm{kV} / \mathrm{m}$ develops. The shape of the profile is dominated by the diamagnetic term.

The causal relationship between radial electric fields and improved confinement was demonstrated in biasing experiments[47]. By means of polariza-
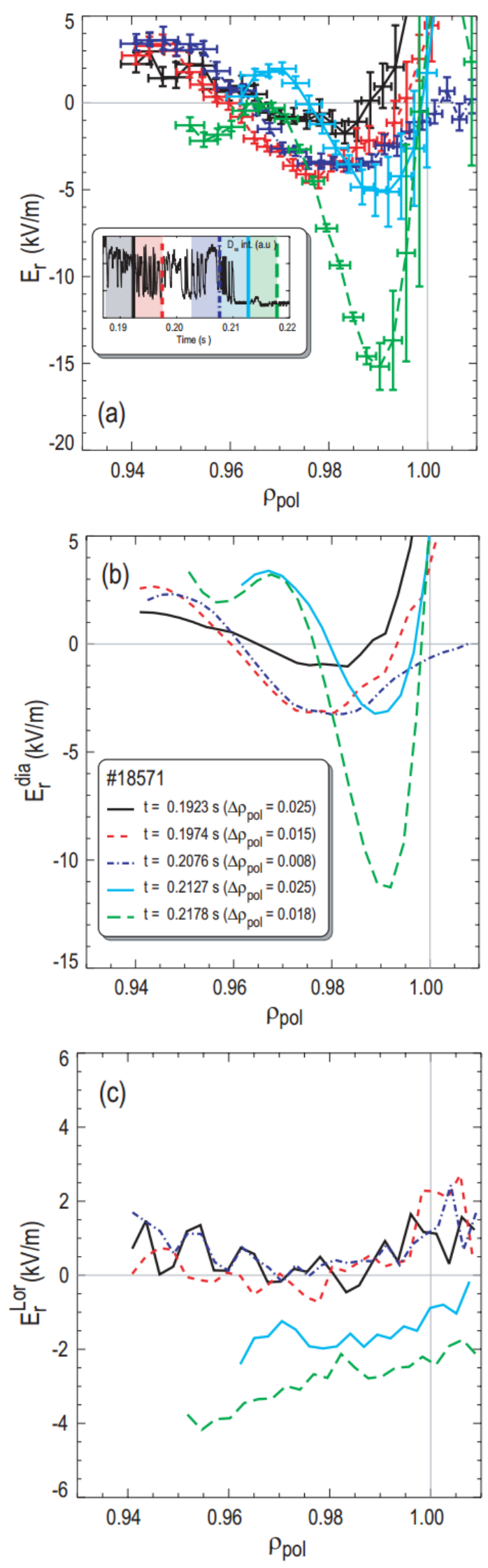

Figure 6: Evolution of $\mathrm{E}_{r}$ through a fast $\mathrm{L} / \mathrm{H}$ transition, (a) total $\mathrm{E}_{r}$, (b) diamagnetic part, (c) Lorentz part (figure taken from [46]) 
tion electrode at the plasma periphery, the edge radial electric field profile could be externally controlled and H-mode transitions could be triggered. Important results were contributed by the TEXTOR tokamak[48, 49].

\section{B. E $\times$ B Shear Effects on internal transport bar-} riers

Internal transport barriers (ITBs) have allowed the reduction of transport coefficients to close to neoclassical levels in a plasma region of finite radial extent, typically around mid-radius. ITBs have been produced on different tokamaks, moreover on DIIID[50], JT-60U[51] and JET[52]. The formation of an ion ITB dramatically reduces ion heat and particle flux from the core (sub-neoclassical ion thermal diffusivity has been obtained). In as much as neoclassical transport is usually considered to be as the minimum transport possible in a tokamak, these results represent a dramatic improvement in confinement and performance. Furthermore, the strong pressure gradient associated with ITBs drives a bootstrap current which can substantially contribute to overcome the limited pulse length in tokamaks.

Most likely ITB dynamics is controlled by a combination of two or more of the following main mechanisms: (1) $\mathbf{E} \times \mathbf{B}$ flow shear ; (2) magnetic shear $s=r / q$ $d q / d r$ and low order rational $q$-surface, (3) the influence of the ratio $\mathrm{T}_{i} / \mathrm{T}_{e}$ or strong electron density gradients (e.g. due to pellet injection) on instability growth rates; and (4) turbulence stabilisation by selfgenerated poloidal $\mathbf{E} \times \mathbf{B}$ zonal flows[53]. Numerous triggering mechanisms have been proposed: ion orbit losses[54], Stringer spin-up[55], critical gradients[56], magnetic shear[57, 58], Reynolds stress[59, 60, 61, 62].

Ion ITBs

JET plasmas are heated mainly by NBI and ICRH. The NBI is oriented in co-current direction and is an important source of toroidal momentum. In most cases ITBs on JET are clearly visible in the ion heat and toroidal momentum channels. During strong ITBs large excursions in poloidal rotation velocity have been observed[63]. Both toroidal and poloidal rotation terms contribute equally to the radial electric field in the region with reduced ion heat transport. The contribution from the diamagnetic term is an order of magnitude lower.

In dedicated experiments[64] the toroidal field (TF) ripple was modified, which changed the $\mathbf{E} \times \mathbf{B}$ rotation of the plasma. It was found that in plasmas with large TF ripple and small $\mathbf{E} \times \mathbf{B}$ rotation, ITBs could still be triggered, but did not develop into strong barriers. Also the poloidal rotation velocity seems to be related to the ITB strength, suggesting that it acts as a positive feedback mechanisms, which helps to sustain the region with reduced transport[65]. Figure 7 shows profiles of ITBs for different ripple amplitudes, shot no. 69670 has the standard TF ripple of $\delta=0.08 \%$, shot no. 69665 has $\delta=0.63 \%$, shot no. 69684 has $\delta=0.82 \%$ and shot no. 69690 has $\delta=1.00 \%$. The $\mathrm{T}_{i}$ and $\omega_{\phi}$ gradients are decreasing with increasing ripple, as is the excursion in $\mathrm{v} \theta$.

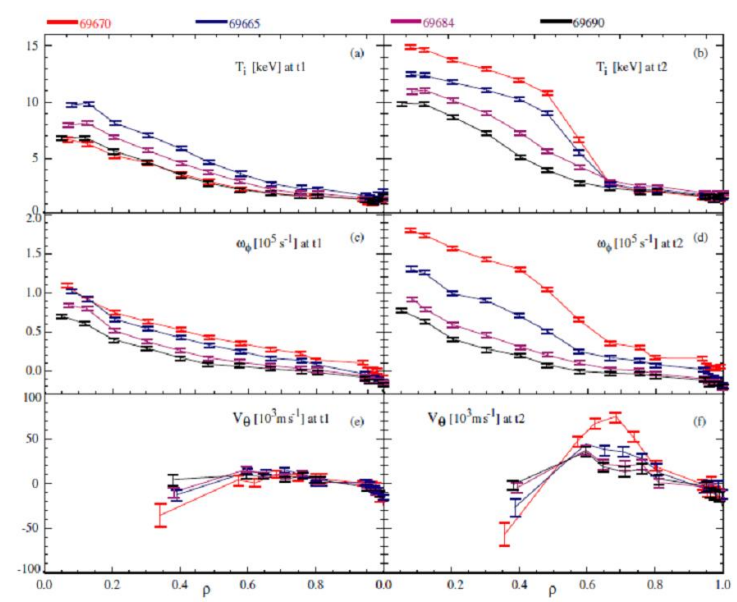

Figure 7: (a) and (b) Ion temperature, (c) and (d) toroidal angular frequency and (e) and (f) poloidal rotation velocity for four shots with different ripple amplitudes and reversed magnetic shear. Profiles on the left hand side are before the ITB. Profiles on the right hand side are during the ITB phase (figure taken from $[65]$ )

In recent $\underline{D I I I-D}$ discharges with varying percentages of co- and counter-injected neutral beam fractions, differences in core barrier formation are observed. For fully co-injected discharges with high toroidal rotation and large $\mathbf{E} \times \mathbf{B}$ shear, either an enduring internal transport barrier (ITB) forms spontaneously or is triggered at the $q_{\text {min }}=2$ crossing. For balanced-injected discharges with low toroidal rotation and small $\mathbf{E} \times \mathbf{B}$ shear, no core barrier forms; however, transient improvements in transport are seen near integer $q_{\text {min }}$ crossings (figure 8). In all cases reductions in fluctuation amplitudes occur near the rational $q_{\min }$ times as well as jumps in poloidal velocity. The observations support the model that zonal flow effects at integer $q_{\text {min }}$ can act as an ITB trigger and sufficient background $\mathbf{E} \times \mathbf{B}$ shear is required for barrier sustainment[66].

\section{Electron ITBs}

In $D I I I-D$ ITB plasmas, large reductions in transport are observed in the ion $\left(\chi_{i}\right)$, angular momentum $\left(\chi_{\phi}\right)$, and sometimes particle $D$ diffusivities, but a similar large reduction in electron heat transport $\left(\chi_{e}\right)$ is often not observed. In low magnetic shear plasmas, $\chi_{e}$ shows little change, and remains well above $\chi_{i}$. However, in some DIII-D discharges with strongly negative magnetic shear, large reductions in $\chi_{e}$ have been observed[67]. The electron temperature profile steepens just inside the ion ITB, indicating the formation of an electron ITB. In this region $\chi_{e}$ may decrease a factor of $3-10$, but remains far above the neoclassical level (figure 9). 

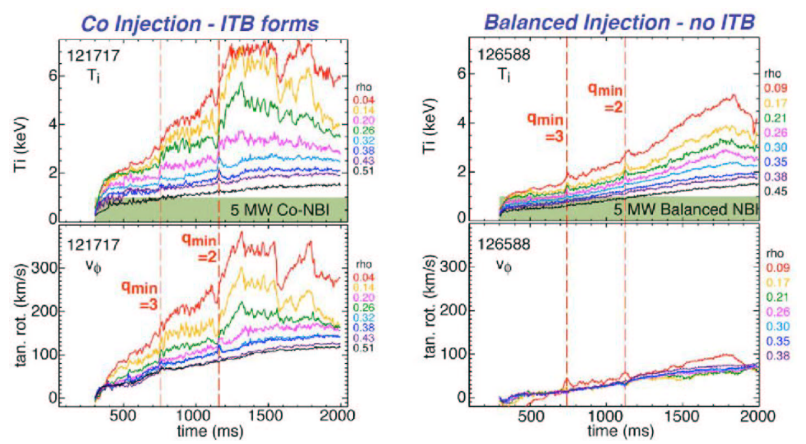

Figure 8: Time traces of ion temperature and toroidal rotation velocity in DIII-D plasmas with co- and balanced NBI-injection. When the $\mathbf{E} \times \mathbf{B}$ shearing rate is low only transient transport improvements are seen when qmin crosses an integer value (figure taken from[66])
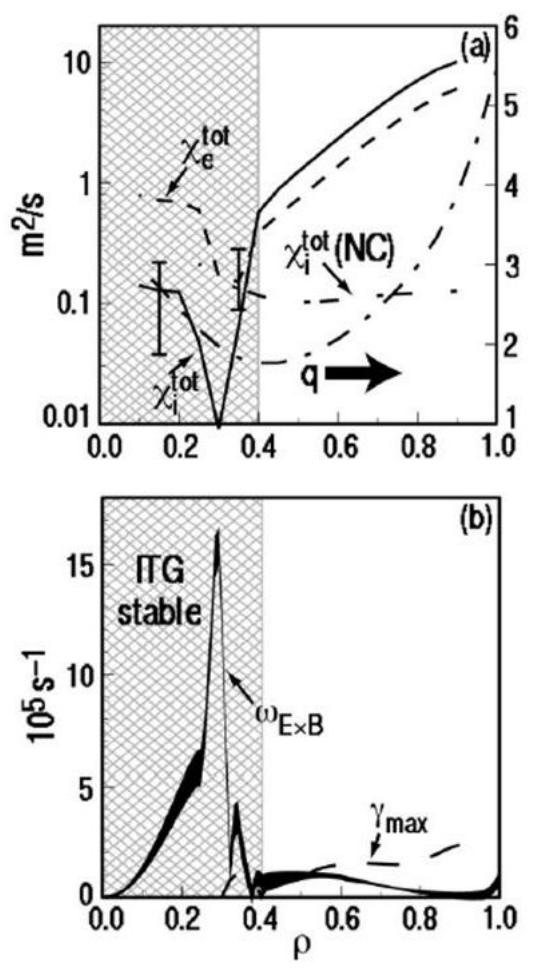

Figure 9: Ion stability in strong negative magnetic shear: (a) ion and electron diffusivity profiles, showing ITB, and $q$ profile and (b) comparison of $\omega_{E x B}$ flow shear rate and predicted $\gamma_{\max }$ for the ITG mode (figure taken from[67]).
The formation of electron ITB was further studied using Electron Cyclotron (EC) heating in JT-60U plasmas with positive (PS) and reversed magnetic shear (RS)[68]. The NBI power was scanned. With no or small NBI power, a strong, box-type electron ITB was formed in RS plasmas while a peaked profile with no strong electron ITB was observed in PS plasmas. Comparison of Gyro Kinetic Simulation predictions with experiments, in low and strongly negative magnetic shear plasmas with an ITB, suggests that the region for improved ion transport seems well characterized by the condition $\omega_{E \times B}>\gamma_{\max }$, where $\omega_{E \times B}$ is the $\mathbf{E} \times \mathbf{B}$ flow shear rate, calculated from measured quantities, and $\gamma_{\max }$ is the maximum calculated linear growth rate for ITG modes in the absence of flow shear. For the electrons, within a limited region just inside the point of ITG mode suppression, the ETG modes appear to dominate the electron thermal transport and, consequently, to provide a lower limit on electron thermal diffusivity. When the NBI power (and thus the shearing rate) was increased in EC-heated PS plasmas, the electron thermal diffusivity was reduced in conjunction with the increase in $E_{r}$ gradient and reduction of ion thermal diffusivity, and strong electron and ion ITBs were formed. When the NBI power was increased in RS plasmas with high power EC heating, in which a strong electron ITB is already established, $\chi_{e}$ was not affected but $\chi_{i}$ decreased and a strong ion ITB was formed with the increase in $E_{r}$ gradient. The dependences of $\chi_{i}$ and $\chi_{e}$ on the shearing rate are shown in figure 10. In this study, it is clearly shown that there is easier access to strong electron ITBs without a large $E_{r}$ gradient in RS plasmas than in PS plasmas. Another new discovery is that electron transport in strong electron ITBs in RS plasmas is not affected by the increase in $E_{r}$ gradient.

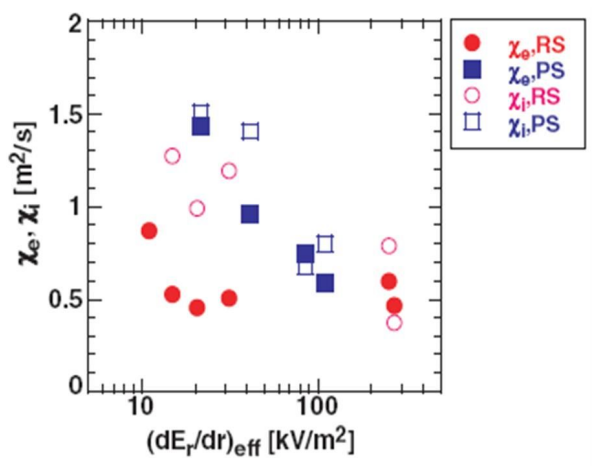

Figure 10: Dependence of minimum values of $\chi_{e}$ and $\chi_{i}$ on the shearing rate. Closed and open circles denote $\chi_{e}$ and $\chi_{i}$ in Reversed Shear plasmas and closed and open squares denote $\chi_{e}$ and $\chi_{i}$ in Positive Shear plasmas (figure taken from[68])

On the National Spherical Torus Experiment (NSTX) electron ITBs have been formed with no $\mathbf{E} \times \mathbf{B}$ shear, by heating only with High Harmonic Fast Wave 
(HHFW) radio frequency heating[69]. It was found that in plasma with strongly negative magnetic shear $(s)$ electron scale fluctuations were suppressed and the $T_{e}$ profiles show a strong transport barrier around the region of minimum $s$ (figure 11). Experiments have been conducted to investigate the interplay between the formation of electron ITBs and the maintenance of self-consistent plasma profiles under the action of Electron Cyclotron Resonance Heating and Current Drive ECRH/ECCD. A joint analysis of

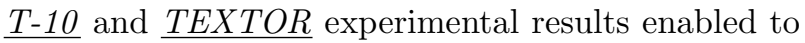
analyse effects bound with plasma self-organization. It was shown that the plasma pressure profiles obtained in different operational regimes and even in various tokamaks may be represented by a single typical curve, called the self-consistent pressure or canonical profile, also often referred to as profile resilience or profile stiffness[70].

Both phenomena, the self consistent profile and ITB, are connected with the density of rational magnetic surfaces, where the turbulent cells are situated. The distance between these cells determines the level of their interaction, and therefore the level of the turbulent transport. This process regulates the plasma pressure profile. If the distance is wide, the turbulent flux may be diminished and the ITB may be formed. In regions with rarefied surfaces the steeper pressure gradients are possible without instantaneously inducing pressure driven instabilities, which force the profiles back to their self-consistent shapes[71].

\section{CONCLUSIONS AND FUTURE STUDIES}

The importance of radial electric fields is now widely recognized. It has been demonstrated in limiter- and divertor tokamaks, helical devices and mirror machines with a variety of discharge- and heating conditions as well as edge biasing schemes that improved confinement is often associated with strong $\mathbf{E} \times \mathbf{B}$ velocity shear. Turbulence stabilisation is a robust and universal mechanism which plays a major role in the formation and sustainment of transport barriers in magnetic confinement devices.

A negative well in the edge electric field profile has been measured in different tokamaks during improved confinement modes. The parameters that determine the shape of the $\mathrm{E}_{r}$ profile may differ from machine to machine, but the depth of the well (and thus the ExB shear) seems to be linked to the level of improved confinement.

Different mechanisms play a role in the triggering and sustainment of internal transport barriers. It has been demonstrated that synergistic effects exist between $\mathbf{E} \times \mathbf{B}$ velocity shear and magnetic shear. It has been found that a stable ion ITB can most easily be created in the vicinity of low order rational $q$ - surfaces when a certain background $\mathbf{E} \times \mathbf{B}$ velocity shear is present $[64,65,66]$. Experiments are ongoing to
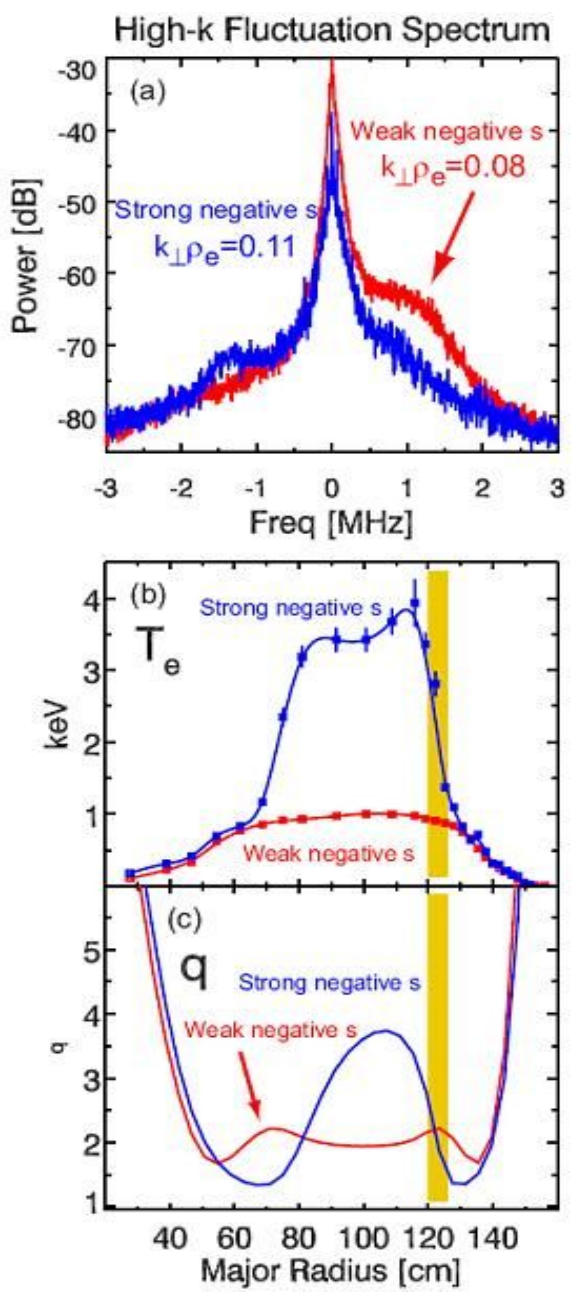

Figure 11: (a) High- $k$ microwave scattering fluctuation power spectra comparison between a case with an e-ITB and strongly negative magnetic shear vs a weakly reversed shear case with lower electron temperature gradients. (b) Electron temperatures and (c) $q$-profiles for cases shown in (a), shaded region indicates the high- $k$ measurement region (figure taken from [69]) 
study the formation of electron ITBs. The hypothesis is that the formation of an electron transport barrier is determined by the density of turbulent cells in the vicinity of low-order rational surfaces, a negative magnetic shear is favourable to a non-reversed $q$-profile.

Further improved comparison between experiment and theory requires the development or improvement of plasma diagnostics with higher spatial and temporal resolution.

\section{REFERENCES}

1. K. CROMBE and G. VAN OOST, Transactions of Fusion Science and Technology 61, $\mathrm{N}^{o} 2 \mathrm{~T}$, (2012)

2. P. HELANDER, "Classical and neoclassical transport in tokamaks", Transactions of Fusion Science and Technology 61, No 2T , (2012)

3. V. ROZHANSKY, Reviews of Plasma Physics 24, p.1, Springer (2008)

4. J. HEIKKINEN et al., Phys. Rev. Letters 84, p.487 (2000)

5. V. ROZHANSKY et al., Contrib. Plasma Physics 40, p.423, Springer (2000)

6. T. BUDKER, in Plasma Physics and the Problem of Controlled Thermonuclear Reactions, edited by M.A. LEONTOVICH, Pergamon Press, New York, 1, p.78 (1951)

7. T. STIX, Phys. Fluids 14, 692 (1971)

8. J.R. ROTH et al., Phys. Rev. Letters 22, 1450 (1978)

9. J.R. ROTH, Proc. IAEA Technical Conference Meeting on Tokamak Plasma Biasing, Montreal (IAEA Vienna), p. 132 (1992)

10. J.G. GORMAN and L.H. RIETJENS, Phys. Fluids 9, 2504 (1966)

11. E.J. STRAIT, Nucl. Fusion 21, 943 (1981)

12. R.J. TAYLOR et al., Plasma Physics and Control. Thermonuclear Research 3 (IAEA Vienna), p. 251 (1982)

13. W7-A team et al., Proc. $3^{\text {rd }}$ Joint VarennaGrenoble , Int. Symp. Heating in Toroidal Plasma, Grenoble 2, p.813 (1982)

14. J.C. HOSEA et al., Phys. Rev. Letters 30, 839 (1973)

15. K.A. RAZUMOVA, Plasma Physics and Control. Fusion 26 (1984) 37
16. M. MURAKAMI et al., in Plasma Physics and Controlled Nuclear Fusion Research, 1 (IAEA Vienna), p. 87 (1984)

17. F. WAGNER et al., Phys. Rev. Letters 49, 1408 (1982)

18. R.J. TAYLOR et al., Phys. Rev. Letters 63, 2365 (1989)

19. R.J. GROEBNER, K.H. BURRELL and R.P. SERAYDARIAN, Phys. Letters 64, 3015 (1990)

20. S.-I. ITOH and K. ITOH, Phys. Rev. Letters, 60, p. 2276 (1988); K. ITOH, S.-I. ITOH, A. FUKUYAMA, "Transport and Structural Formation in Plasma", I.O.P. Publishing, Bristol (1999)

21. K.C. SHAING, E.C. CRUME Jr. And W.A. HOUlBERG, Phys. Rev. Letters 63, 2369 (1989)

22. M. TENDLER, Plasma Physics and Controlled Fusion 39, B371 (1997).

23. R.R. WEYNANTS et al, Proc. $17^{\text {th }}$ Eur. Conf. On Controlled Fusion and Plasma Physics, Amsterdam, 1, (Europhysics Conf. Abstr. 14B), p. 287 (1990).

24. R.R. WEYNANTS et al, Nucl. Fusion 32, p. 837 (1992).

25. R.R. WEYNANTS and G. VAN OOST, Plasma Physics and Controlled Fusion 35, B177 (1993).

26. G. VAN OOST et al., Plasma Physics and Controlled Fusion 45,621 (2003), and references therein.

27. K.H. BURRELL, Phys. Plasmas 4, 1499 (1997)

28. D. HARTMANN, "Stellarators", Transactions of Fusion Science and Technology 61, $\mathrm{N}^{o} 2 \mathrm{~T}$, (2012)

29. K. IDA, Plasma Physics and Controlled Fusion 40, 1429 (1998).

30. Proceedings of the Technical Committee Meeting on H-mode Physics, Kloster Seeon, Germany, September 22-24, 1997, in Plasma Physics and Controlled Fusion 40, Nr.5.

31. G. VAN OOST and M. TENDLER, Plasma Physics and Controlled Fusion 44, 1761 (2002).

32. T.S. HAHM and K.H. BURRELL, Phys. Plasmas 2, 1648 (1995).

33. K.H. BURRELL, Plasma Physics and Control Fusion 36, A291 (1994). 
34. A.J.H. DONNÉ, A.V. MELNIKOV, G. VAN OOST, Czechoslovak J. of Physics 52, 1077(2002).

35. R. JASPERS, "Spectroscopy", Transactions of Fusion Science and Technology 61, $\mathrm{N}^{o} 2 \mathrm{~T}$, (2012).

36. Special issue on heavy ion beam probing, IEEE Trans. on Plasma Science 22, Nr. 4 (1994).

37. G. VAN OOST, "Advanced probes for boundary plasma diagnosis in fusion devices", Transactions of Fusion Science and Technology 61, $\mathrm{N}^{o} 2 \mathrm{~T}$, (2012).

38. C. MAGGI et al., Nucl. Fusion 54 , p. 023007 (2014)

39. B.W. RICE et al., Phys. Rev.Letters Vol. 79, No. 14 (1997), 2694-2697

40. F. WAGNER et al., Plasma Phys. Control. Fusion 49 (2007) B1-B33

41. E. VIEZZER et al., Nucl. Fusion 53 , p. 053005 (2013)

42. P. SAUTER et al., Nucl. Fusion 52 , p. 012001 (2012)

43. Y. ANDREW et al., Europhysics Letters 83 (2008) 15003

44. R.M. MCDERMOTT et al., Physics of Plasmas 16 (2009) 056103

45. J. SCHIRMER et al., Nuclear Fusion 46 (2006) S780

46. H. MEYER et al., Journal of Physics, Conference Series 123 (2008) 012005

47. G. VAN OOST et al., Czech. J. Phys. 51 (2001) 957

48. R.R. WEYNANTS et al., Nuclear Fusion 32 (1992) 837

49. J. BOEDO et al., Nucl. Fusion 40 (2000) 1397

50. E. LAZARUS et al., Phys. Rev. Letters 77, 2714 (1996).

51. K. USHIGUSA and the JT-60 team, Plasma Physics and Controlled Nuclear Fusion Research, 1 (IAEA Vienna) 37 (1996).

52. C. GORMEZANO, in Plasma Physics and Controlled Fusion 41, B367 (1999).

53. Special issue on Experimental studies of zonal flow and turbulence, Plasma Phys. Control. Fusion 48, 1181 (2006).
54. K.C. SHAING et al., Phys. Rev. Letters 63 (1989), 2369

55. A.B. HASSAM et al., Phys. Rev. Letters 66 (1991) 309

56. F.L. HINTON and G.M. STAEBLER Phys. Fluids B 5 (1993) 1281

57. C. KESSEL et al. Phys. Rev. Letter 72 (1994), 1212

58. E. SYNAKOWSKI Phys. Plasmas 4 (1994), 1736

59. P. DIAMOND, Y. KIM, Phys.Fluids B 3 (1991) 1626

60. B.A. CARRERAS et al Phys.Fluids B 3 (1991) 1438

61. P. DIAMOND et al Phys. Rev. Letters $\mathbf{7 2}$ (1994), 2565

62. C. HIDALGO et al., Plasma Phys. Control. Fusion 42 (2000) A153-A160

63. K. CROMBÉ et al., Phys.Rev.Lett. 95,155003 (2005)

64. P.C. DE VRIES et al., Plasma Phys. Control. Fusion 50 (2008) 065008

65. K. CROMBÉ et al., Plasma Phys. Control. Fusion 51 (2009) 055005

66. M.E. AUSTIN et al. "The Effect of $\boldsymbol{E} \times \boldsymbol{B}$ Shear on Core Barrier Formation Near Low-Order Rational q Surfaces" presented at the joint EU-US Transport Taskforce Workshop: San Diego, California April 28-May 1, 2009

67. B.W. STALLARD et al., Phys. Plasmas 6 (1999)

68. T. FUJITA et al., Plasma Phys. Control. Fusion 46 (2004) A35-A43

69. H.Y. YUH et al., Phys. Plasmas 16 (2009), 056120

70. Y.N. DNESTROVSKI et al., Nuclear Fusion 46 953 (2006) and references therein.

71. K.A. RAZUMOVA et al., Plasma Phys. Control. Fusion 48, 1373 (2006).

72. A.V. MELNIKOV et al., Nuclear Fusion $\mathbf{5 1}$ (2011) 083043 